\title{
Clustering with EM: Complex Models vs. Robust Estimation
}

\author{
C. Saint-Jean, C. Frélicot, and B. Vachon \\ L3I - UPRES EA 1216 \\ Avenue de Marillac,17042 La Rochelle Cedex 1 \\ \{csaintje, cfrelico, bvachon\}@univ-lr.fr
}

\begin{abstract}
Clustering multivariate data that are contaminated by noise is a complex issue, particularly in the framework of mixture model estimation because noisy data can significantly affect the parameters estimates. This paper addresses this problem with respect to likelihood maximization using the Expectation-Maximization algorithm. Two different approaches are compared. The first one consists in defining mixture models that take into account noise. The second one is based of robust estimation of the model parameters in the maximization step of EM. Both have been tested separately, then jointly. Finally, a hybrid model is proposed. Results on artificial data are given and discussed.
\end{abstract}

Keywords: Clustering, Expectation-Maximization, Robustness, M-estimation

\section{Introduction}

Clustering techniques are successfully applied in many areas and some software can be now downloaded from the Internet, e.g. EMMIX by G. McLachlan and al. 4], MCLUST by C. Fraley and A. Raftery [5]. It aims at describing relationships between objects in order to group them in homogeneous clusters. Let $\chi=x_{1}, x_{2}, \ldots, x_{N}$ be an observed $p$-dimensional random sample of size $N$. In mixture model theory, each $x_{k}(k=1, N)$ is assumed to be a realization of a $p$-dimensional random vector $X$ with the $C$-components mixture probability density function $(p d f)$ :

$$
f(x ; \Theta)=\sum_{i=1}^{C} \pi_{i} f\left(x ; \Theta_{i}\right)
$$

where $f\left(x ; \Theta_{i}\right)$ denotes the $p$-dimensional pdf of the $i^{\text {th }}$ component and pairs $\left(\pi_{i}, \Theta_{i}\right)(i=1, C)$ are the model parameters. A priori probabilities $\pi_{i}$ sum up to one. If a normal mixture model is assumed, $\Theta_{i}=\left(\mu_{i}, \Sigma_{i}\right)^{T}$ with mean $\mu_{i}$ and covariance matrix $\Sigma_{i}$. Assuming independent features of $X$, the model parameters $\Theta=\left(\pi_{1}, \ldots, \pi_{C}, \Theta_{1}^{T}, \ldots, \Theta_{C}^{T}\right)^{T}$ can be estimated by maximizing the likelihood $\mathcal{L}(\Theta)$ using the Expectation-Maximization (EM) iterative algorithm 
due to Dempster, Laird and Rubin [3]:

$$
\mathcal{L}(\Theta)=P(\chi \mid \Theta)=\prod_{k=1}^{N} \sum_{i=1}^{C} \pi_{i} f\left(x_{k} ; \Theta_{i}\right)
$$

Resulting clustering is not robust, i.e. it is too much sensitive to outliers or noise. In this paper, we address the problem of clustering noisy data. To face such a problem, one can choose either to perform robust estimates or to use more complex mixture models. In section 2, we briefly recall the EM algorithm. Robust M-estimates that can be used are presented in section 3; and we focus on the normal case. Next, we present a comparative study of both strategies on artificial two-dimensional data.

\section{EM Algorithm}

In clustering problems, observed data can be regarded as being incomplete data because the labelling is unknown. Complete data $y_{k}=\left(x_{k}, z_{k}\right)$ can be defined by introducing for all observation $x_{k}$ the realization $z_{k}=\left(z_{k 1}, \ldots, z_{k C}\right)$ of a Cdimensional random variable $Z$ representing the labels of $x_{k}$, i.e. $z_{k i}$ is equal to 1 when $x_{k}$ arises from the $i^{t h}$ component and 0 otherwise. Then, the maximization of the likelihood (2) can be replaced by an easier one, namely the complete likelihood $\mathcal{L}_{c}(\Theta)=P(X, Z \mid \Theta)$ maximization. This is achieved by the EM algorithm that performs iterative maximization of the complete-data likelihood expectation:

$$
Q\left(\Theta ; \Theta^{(t)}\right)=E\left[\mathcal{L}_{c}(\Theta) \mid \chi, \Theta^{(t)}\right]
$$

where $(t)$ is an iteration index. Each EM iteration consists of two steps. Computation of $Q\left(\Theta ; \Theta^{(t)}\right)$ corresponds to the so-called E-Step (Expectation Step). Assuming independent $z_{k}$, the complete-data log-likelihood is:

$$
\log \left(\mathcal{L}_{c}(\Theta)\right)=\sum_{k=1}^{N} \sum_{i=1}^{C} z_{k i} \log \left(\pi_{i} f\left(x_{k} ; \Theta_{i}\right)\right)
$$

Therefore:

$$
Q\left(\Theta ; \Theta^{(t)}\right)=\sum_{k=1}^{N} \sum_{i=1}^{C} E\left[z_{k i} \mid \chi, \Theta^{(t)}\right] \log \left(\pi_{i} f\left(x_{k} ; \Theta_{i}\right)\right)
$$

and the E-Step reduces to estimating $E\left[z_{k i} \mid \chi, \Theta^{(t)}\right]$. Let $\hat{z}_{k i}$ be this estimate. The second step M-Step (Maximization Step) of EM consists in finding the value of $\Theta$ that maximizes $Q\left(\Theta ; \Theta^{(t)}\right)$ :

$$
\Theta^{(t+1)}=\arg \max _{\Theta} Q\left(\Theta ; \Theta^{(t)}\right)
$$

The EM algorithm increases monotonically the likelihood (see [9] by C.F.J. Wu for details). 
When a $p$-dimensional normal mixture model is assumed, the parameters of each component $\left(\mu_{i}, \Sigma_{i}\right)$ as well as the prior probability $\pi_{i}$ are iteratively estimated using $\hat{z}_{k i}=\frac{\pi_{i} f\left(x_{k} ; \Theta_{i}\right)}{\sum_{j=1}^{C} \pi_{j} f\left(x_{k} ; \Theta_{j}\right)}$ :

$$
\begin{gathered}
\hat{\pi}_{i}^{(t+1)}=\frac{\sum_{k=1}^{N} \hat{z}_{k i}}{N} \\
\hat{\mu}_{i}^{(t+1)}=\frac{\sum_{k=1}^{N} \hat{z}_{k i} x_{i}}{\sum_{k=1}^{N} \hat{z}_{k i}} \\
\hat{\Sigma}_{i}^{(t+1)}=\frac{\sum_{k=1}^{N} \hat{z}_{k i}\left(x_{k}-\hat{\mu}_{i}^{(t+1)}\right)\left(x_{k}-\hat{\mu}_{i}^{(t+1)}\right)^{T}}{\sum_{k=1}^{N} \hat{z}_{k i}}
\end{gathered}
$$

\section{Robust Estimation}

Let $a=\left(a_{1}, \ldots, a_{m}\right)$ be a parameter to be estimated within a sample. Let $e_{k}$ be the difference between an observed $x_{k}$ and its predicted value $\hat{x}_{k}$, namely an error. $e_{k}$ is a realization on a random variable $e$ whose probability distribution is $J$. Assuming independent samples, the likelihood to be maximized is a product. Optimal $a$ can be obtained by minimizing the following cost function:

$$
C(a)=\sum_{k=1}^{N} \rho\left(\frac{x_{k}-\hat{x}_{k}(a)}{\sigma_{k}}\right)
$$

where $\rho=\log \left(J^{-1}\right)$ and $\sigma_{k}$ is a weighting factor. This is achieved by solving the differential equations:

$$
\frac{\partial C(a)}{\partial a_{j}}=\sum_{k=1}^{N} \frac{1}{\sigma_{k}} \psi\left(\frac{x_{k}-\hat{x}_{k}(a)}{\sigma_{k}}\right) \frac{\partial \hat{x}_{k}(a)}{\partial a_{j}}=0
$$

where $\psi(x)=\frac{d \rho}{d x}(x)$. Different M-estimate models are shown in Table 1 where $w(x)=\frac{\psi(x)}{x}$ is a weight function increasing as the error decreases.

Table 1. Different M-estimates

\begin{tabular}{lccc}
\hline Model & $\rho(x)$ & $\psi(x)$ & $w(x)$ \\
\hline $\begin{array}{l}\text { Legendre } \\
\text { Median }\end{array}$ & $x^{2}$ & $2 x$ & 2 \\
Cauchy & $\frac{c^{2}}{2} \log \left(1+\left(\frac{x}{c}\right)^{2}\right)$ & $\frac{x}{1+\left(\frac{x}{c}\right)^{2}}$ & $\frac{1}{|x|}$ \\
Huber [6] & $\left\{\begin{array}{l}\frac{x^{2}}{2} \text { if }|x| \leq c \\
c|x|-\frac{c^{2}}{2} \text { else }\end{array}\right.$ & $\left\{\begin{array}{l}x \text { if }|x| \leq c \\
c \text { sgn }(x) \text { else }\end{array}\right.$ & $\left\{\begin{array}{l}1 \text { if }|x| \leq c \\
\frac{c}{|x|} \text { else }\end{array}\right.$ \\
\hline
\end{tabular}


Such models can be used when estimating the parameters $\left(\mu_{i}, \Sigma_{i}\right)$ of the components of a normal mixture by EM [1]. Equation (8) is simply replaced by the following procedure:

$$
\begin{aligned}
& -\tau=0 \\
& \tilde{\mu}_{i}^{(t+1, \tau)}=\hat{\mu}_{i}^{(t+1)} \\
& \tilde{\Sigma}_{i}^{(t+1, \tau)}=\hat{\Sigma}_{i}^{(t+1)} \\
& \text { - repeat } \forall k=1, N: e_{k}=\left(x_{k}-\tilde{\mu}_{i}^{(t+1, \tau)}\right)^{T} \tilde{\Sigma}_{i}^{-1(t+1, \tau)}\left(x_{k}-\tilde{\mu}_{i}^{(t+1, \tau)}\right) \\
& w\left(e_{k}\right)=\frac{\psi\left(e_{k}\right)}{e_{k}} \\
& \tilde{\mu}_{i}^{(t+1, \tau)}=\frac{\sum_{k=1}^{N} w\left(e_{k}\right) \hat{z}_{k i} x_{k}}{\sum_{k=1}^{N} w\left(e_{k}\right) \hat{z}_{k i}} \\
& \tilde{\Sigma}_{i}^{(t+1, \tau)}=\frac{\sum_{k=1}^{N} w\left(e_{k}\right) \hat{z}_{k i}\left(x_{k}-\tilde{\mu}_{i}^{(t+1, \tau)}\right)\left(x_{k}-\tilde{\mu}_{i}^{(t+1, \tau)}\right)^{T}}{\sum_{k=1}^{N} w\left(e_{k}\right) \hat{z}_{k i}} \\
& \tau \leftarrow \tau+1
\end{aligned}
$$

until reached bound

It is worthy of note that the property of monotonous increase of the likelihood is lost by EM in case of robust estimation. The model parameters are no more updated by solving $\frac{d Q\left(\Theta ; \Theta^{(t)}\right)}{d \Theta}=0$. However, these estimates (7) - (9) are good initial values for the robust ones (12) - (13).

Since the weight functions $w$ are monotonous decreasing functions of the error $e_{k}$, the more $\tau$, the more robust but the less precise estimates are provided. Therefore the number of iterations is bounded, e.g.:

$$
\begin{aligned}
& -\tau<\tau_{\max } \\
& -\frac{\left|\tilde{\mu}_{i}^{(t+1, \tau+1)}-\tilde{\mu}_{i}^{(t+1, \tau)}\right|}{\tilde{\mu}_{i}^{(t+1, \tau)}}<\epsilon \\
& - \text { rate of samples having a quite zero weight } \alpha<\alpha_{\max }
\end{aligned}
$$

We have combined the first and the third conditions in our experiments.

\section{Mixture Models}

In this study, we have used normal and uniform components for computation convenience. Let $\mathcal{N}$ denotes the p-dimensional gaussian pdf and $\mathcal{U}$ the uniform one defined on a given hypercube $H$. Here are the more or less complex mixture models that we have tested:

1. $C$ normal components where the parameters are estimated via (7) - (9):

$$
f(x ; \Theta)=\sum_{i=1}^{C} \pi_{i} \mathcal{N}\left(x ; \mu_{i}, \Sigma_{i}\right)
$$


2. $C$ normal and one uniform components, $\gamma$ being user defined:

$$
f(x ; \Theta)=\gamma \mathcal{U}(x ; H)+\sum_{i=1}^{C} \pi_{i} \mathcal{N}\left(x ; \mu_{i}, \Sigma_{i}\right)
$$

3. $C$ normal components where robust estimates (12) - (13) are used:

$$
f(x ; \Theta)=\sum_{i=1}^{C} \pi_{i} \mathcal{N}\left(x ; \mu_{i}, \Sigma_{i}\right)
$$

4. $C$ normal and one uniform components with robust estimates:

$$
f(x ; \Theta)=\gamma \mathcal{U}(x ; H)+\sum_{i=1}^{C} \pi_{i} \mathcal{N}\left(x ; \mu_{i}, \Sigma_{i}\right)
$$

5. $C$ normal components with robust estimates and one additional mixture:

$$
\begin{aligned}
f(x ; \Theta) & =\sum_{i=1}^{C+1} \pi_{i} f\left(x ; \Theta_{i}\right) \\
f\left(x ; \Theta_{i}\right) & =\left(1-\gamma_{i}\right) \mathcal{N}\left(x ; \mu_{i}, \Sigma_{i}\right) \quad \forall i=1, C \\
f\left(x ; \Theta_{C+1}\right) & =\sum_{i=1}^{C} \gamma_{i} \mathcal{N}\left(x ; \mu_{i}, \alpha_{i} \Sigma_{i}\right)
\end{aligned}
$$

In this latter model we propose, each of the $C$ components is a linear combination of two normal pdf. The first one intends to track cluster kernel points while the second one is supposed to deal with surrounding outliers via multiplicative coefficients $\alpha_{i}$. All these second modes are summed up to compose a $(C+1)^{t h}$ component. The combination coefficients $\gamma_{i}$ are user-defined as well as $\alpha_{i}$.

Our model differs from previous work, e.g. G. Mac Lachlan and D. Peel [8] or Y. Kharin [7], in mixing robust estimation of the parameters in (19) and classical estimation of ones in 20 .

\section{Experiments}

We have generated two data sets in order to test the robustness of the presented mixture models. Both consist of three 2-dimensional gaussian classes and uniformly distributed samples supposed to be noisy points, as shown on figure 1 . In the first set the classes are well-separated while they strongly overlap in the second one. Noisy patterns represent respectively $30 \%$ and $16.7 \%$ of each data set. Table 2 summarizes the theoretical parameters of the classes for both generated datasets. The correlation coefficients $r_{i}$ that describe the clusters orientations are given. As most of partitioning methods for clustering data, the number of 

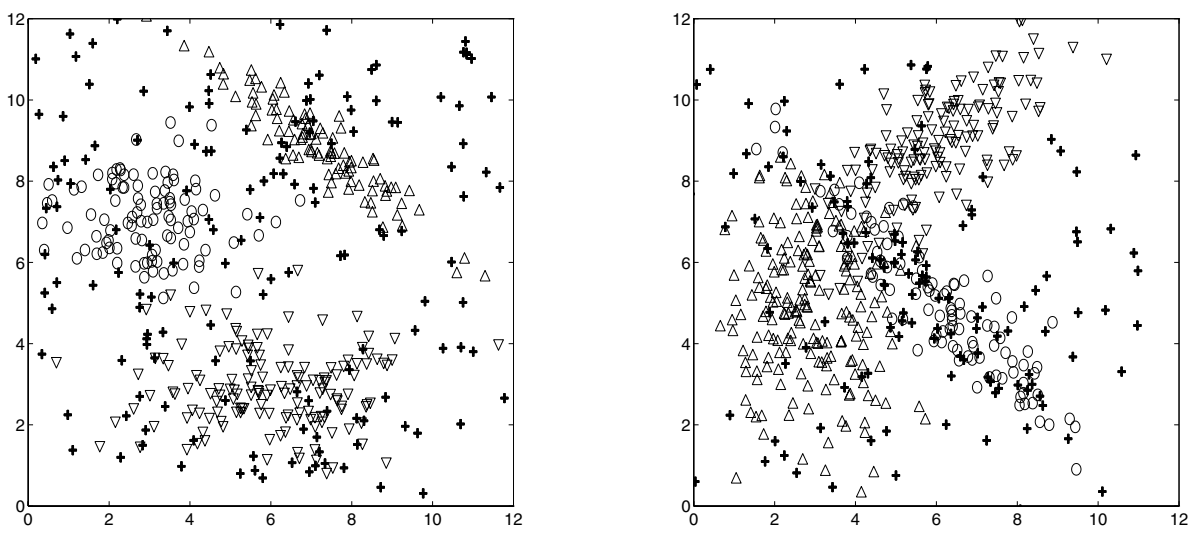

Fig. 1. Data sets \#1 (left) and \#2 (right)

mixture components has to be set. One can assess this value [2] but we preferred to choose it manually $(C=3)$ in order to concentrate on robust estimation effect. The fitted parameters provided by EM significantly depend on initial values. For each model and each data set, we have run EM 50 times with same random initial values $\hat{\mu}_{i}$ and identity matrices for covariance matrices. We also have tried different values of the coefficients involved in the different models and kept the best results according to the maximum posterior probability criterion for the only gaussian points. Only the Cauchy M-estimate has been tested. In addition, the resulting cluster correlation coefficients $r_{i}$ are estimated.

Not surprisingly, all the models give a similar low error rate value on data set \#1 (see Table 3). As the gaussian classes are well separated and as the noise rate is low enough $(30 \%)$ the optimal error rate has been obtained for a low value of the mixing coefficients $\gamma$ or $\gamma_{i}$ (models \#2,\#4 or \#5). Therefore, robust estimation has a not a significant action. The Cauchy parameter $c$ whose value

Table 2. Data sets parameters

\begin{tabular}{|l|lll|lll|lll|}
\hline & $\mu_{1}$ & $\Sigma_{1}$ & $r_{1}$ & $\mu_{2}$ & $\Sigma_{2}$ & $r_{2}$ & $\mu_{3}$ & $\Sigma_{3}$ & $r_{3}$ \\
\hline Data set \#1 & $\left(\begin{array}{l}7 \\
9\end{array}\right)$ & $\left(\begin{array}{cc}3 & -2 \\
-2 & 1.5\end{array}\right)$ & $\frac{-2 \sqrt{2}}{3}$ & $\left(\begin{array}{l}6 \\
3\end{array}\right)$ & $\left(\begin{array}{ll}3 & 0 \\
0 & 1\end{array}\right)$ & 0 & $\left(\begin{array}{l}3 \\
7\end{array}\right)$ & $\left(\begin{array}{ll}1 & 0 \\
0 & 1\end{array}\right)$ & 0 \\
\hline Data set \#2 & $\left(\begin{array}{l}3 \\
5\end{array}\right)$ & $\left(\begin{array}{ll}1 & 0 \\
0 & 3\end{array}\right)$ & 0 & $\left(\begin{array}{l}6 \\
9\end{array}\right)$ & $\left(\begin{array}{cc}2 & 1.5 \\
1.5 & 2\end{array}\right)$ & $\frac{3}{4}$ & $\left(\begin{array}{l}6 \\
5\end{array}\right)$ & $\left(\begin{array}{cc}3.25 & -3 \\
-3 & 3.25\end{array}\right)$ & $\frac{-12}{13}$ \\
\hline
\end{tabular}


Table 3. Data set \#1 - Results (final estimates values and errors)

\begin{tabular}{|c|c|c|c|c|c|}
\hline & Model \#1 & Model \#2 & Model \#3 & Model \#4 & Model \#5 \\
\hline$\mu_{1}$ & $\left(\begin{array}{l}7.07 \\
8.91\end{array}\right)$ & $\left(\begin{array}{l}7.18 \\
8.87\end{array}\right)$ & $\left(\begin{array}{l}7.17 \\
8.86\end{array}\right)$ & $\left(\begin{array}{l}7.14 \\
8.84\end{array}\right)$ & $\left(\begin{array}{l}7.16 \\
8.86\end{array}\right)$ \\
\hline$\Sigma_{1}$ & $\left(\begin{array}{cc}5.32 & -1.69 \\
-1.69 & 2.28\end{array}\right)$ & $\left(\begin{array}{cc}2.49 & -1.59 \\
-1.59 & 1.56\end{array}\right)$ & $\left(\begin{array}{cc}0.89 & -0.63 \\
-0.63 & 0.59\end{array}\right)$ & $\left(\begin{array}{cc}1.72 & -1.2 \\
-1.2 & 1.17\end{array}\right)$ & $\left(\begin{array}{cc}2.77 & -1.36 \\
-1.36 & 1.47\end{array}\right)$ \\
\hline$r_{1}$ & -0.48 & -0.81 & -0.87 & -0.87 & -0.67 \\
\hline$\mu_{2}$ & $\left(\begin{array}{l}6.07 \\
2.74\end{array}\right)$ & $\left(\begin{array}{l}6.12 \\
2.73\end{array}\right)$ & $\left(\begin{array}{l}6.21 \\
2.73\end{array}\right)$ & $\left(\begin{array}{l}6.09 \\
2.73\end{array}\right)$ & $\left(\begin{array}{l}6.09 \\
2.73\end{array}\right)$ \\
\hline$\Sigma_{2}$ & $\left(\begin{array}{cc}4.46 & -0.01 \\
-0.01 & 1.13\end{array}\right)$ & $\left(\begin{array}{ll}4.06 & 0.08 \\
0.08 & 1.04\end{array}\right)$ & $\left(\begin{array}{cc}1.77 & -0.01 \\
-0.01 & 0.41\end{array}\right)$ & $\left(\begin{array}{ll}3.31 & 0.00 \\
0.00 & 0.89\end{array}\right)$ & $\left(\begin{array}{cc}3.45 & -0.00 \\
-0.00 & 0.90\end{array}\right)$ \\
\hline$r_{2}$ & -0.00 & 0.04 & -0.01 & -0.00 & -0.00 \\
\hline$\mu_{3}$ & $\left(\begin{array}{l}2.77 \\
6.90\end{array}\right)$ & $\left(\begin{array}{l}2.98 \\
7.00\end{array}\right)$ & $\left(\begin{array}{l}2.99 \\
7.06\end{array}\right)$ & $\left(\begin{array}{l}2.97 \\
7.03\end{array}\right)$ & $\left(\begin{array}{l}2.86 \\
6.99\end{array}\right)$ \\
\hline$\Sigma_{3}$ & $\left(\begin{array}{cc}1.72 & -0.38 \\
-0.38 & 1.26\end{array}\right)$ & $\left(\begin{array}{cc}2.05 & -0.37 \\
-0.37 & 1.66\end{array}\right)$ & $\left(\begin{array}{cc}1.03 & -0.08 \\
-0.08 & 0.76\end{array}\right)$ & $\left(\begin{array}{cc}1.78 & -0.2 \\
-0.2 & 1.22\end{array}\right)$ & $\left(\begin{array}{cc}1.56 & -0.31 \\
-0.31 & 1.12\end{array}\right)$ \\
\hline$r_{3}$ & -0.26 & -0.20 & -0.09 & -0.13 & -0.23 \\
\hline $\mathrm{E}$ & $3.43 \%$ & $3.43 \%$ & $2 . \%$ & $3.43 \%$ & $2.86 \%$ \\
\hline
\end{tabular}

describes the number of observed points contributing to the robust estimates compensates a low value of the mixing coefficients (if used). The smaller $\gamma$ or $\gamma_{i}$ are, the less noisy points are modelled. So the smaller Cauchy's parameter c is in order to filter enough. The means are very close to the theoretical ones whatever the model is. On the other hand, taking the noise into account (models \#2,\#3,\#4 and \#5) clearly improve the clusters shapes and orientations as reflected by the obtained covariance matrices and correlation coefficients. The optimal partition we obtained with model \#3 is shown on Figure 2 (left hand-side). Obviously,
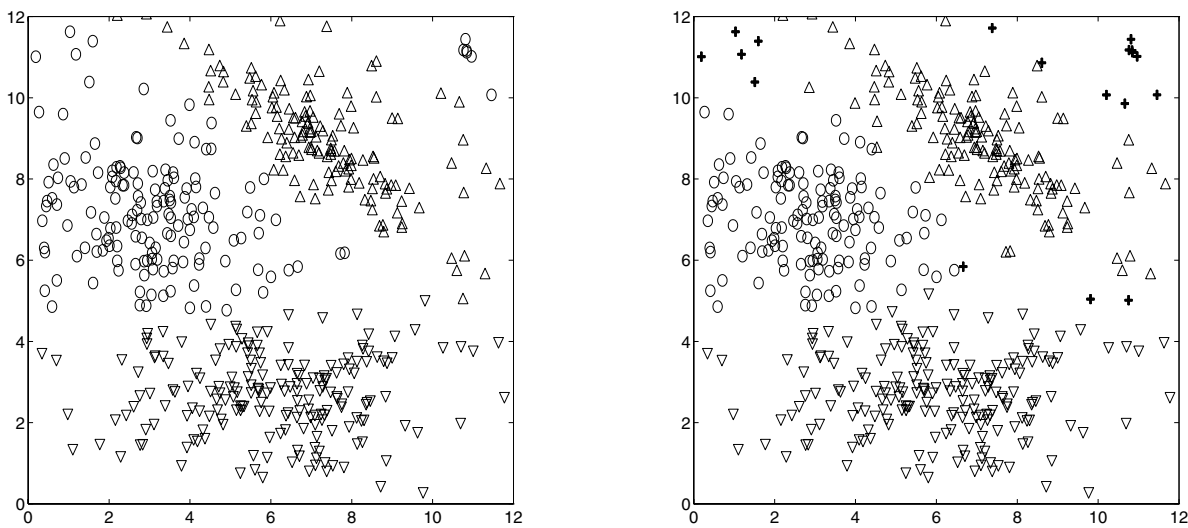

Fig. 2. Data set \#1 clustering with models \#3 (left) and \#5 (right) 
all the outliers have been incorrectly clustered, the few ones in the upper right corner in particular. This can be explained by the fact that original noisy points do not enter into the error rate computation. Clusters resulting from the model we propose (\#5) are shown on Figure 2 (right hand-side).

Table 4. Data set \#2 - Results (final estimates values and errors)

\begin{tabular}{|c|c|c|c|c|c|}
\hline & Model \#1 & Model \#2 & Model \#3 & Model \#4 & Model \#5 \\
\hline$\mu_{1}$ & $\left(\begin{array}{l}3.97 \\
5.06\end{array}\right)$ & $\left(\begin{array}{l}2.84 \\
4.65\end{array}\right)$ & $\left(\begin{array}{l}2.74 \\
4.57\end{array}\right)$ & $\left(\begin{array}{l}3.02 \\
5.20\end{array}\right)$ & $\left(\begin{array}{l}2.95 \\
5.11\end{array}\right)$ \\
\hline$\Sigma_{1}$ & $\left(\begin{array}{ll}5.60 & 0.03 \\
0.03 & 4.54\end{array}\right)$ & $\left(\begin{array}{cc}1.45 & -0.38 \\
-0.38 & 3.14\end{array}\right)$ & $\left(\begin{array}{cc}0.96 & -0.37 \\
-0.37 & 1.85\end{array}\right)$ & $\left(\begin{array}{cc}1.26 & -0.10 \\
-0.10 & 3.38\end{array}\right)$ & $\left(\begin{array}{cc}1.21 & -0.17 \\
-0.17 & 3.06\end{array}\right)$ \\
\hline$r_{1}$ & 0.01 & -0.18 & -0.28 & -0.05 & -0.09 \\
\hline$\mu_{2}$ & $\left(\begin{array}{l}6.06 \\
9.13\end{array}\right)$ & $\left(\begin{array}{l}5.99 \\
8.94\end{array}\right)$ & $\left(\begin{array}{l}6.03 \\
9.04\end{array}\right)$ & $\left(\begin{array}{l}6.09 \\
9.05\end{array}\right)$ & $\left(\begin{array}{l}6.10 \\
9.06\end{array}\right)$ \\
\hline$\Sigma_{2}$ & $\left(\begin{array}{ll}2.02 & 1.19 \\
1.19 & 1.41\end{array}\right)$ & $\left(\begin{array}{ll}1.70 & 0.95 \\
0.95 & 1.40\end{array}\right)$ & $\left(\begin{array}{ll}1.07 & 0.50 \\
0.50 & 0.81\end{array}\right)$ & $\left(\begin{array}{ll}1.29 & 0.69 \\
0.69 & 1.07\end{array}\right)$ & $\left(\begin{array}{ll}1.24 & 0.56 \\
0.56 & 0.95\end{array}\right)$ \\
\hline$r_{2}$ & 0.70 & 0.61 & 0.53 & 0.59 & 0.52 \\
\hline$\mu_{3}$ & $\left(\begin{array}{l}5.68 \\
5.17\end{array}\right)$ & $\left(\begin{array}{l}5.61 \\
5.30\end{array}\right)$ & $\left(\begin{array}{l}5.45 \\
5.45\end{array}\right)$ & $\left(\begin{array}{l}6.40 \\
4.65\end{array}\right)$ & $\left(\begin{array}{l}6.30 \\
4.74\end{array}\right)$ \\
\hline$\Sigma_{3}$ & $\left(\begin{array}{cc}5.58 & -5.4 \\
-5.4 & 5.53\end{array}\right)$ & $\left(\begin{array}{cc}3.80 & -3.37 \\
-3.37 & 3.45\end{array}\right)$ & $\left(\begin{array}{cc}3.00 & -2.65 \\
-2.65 & 2.70\end{array}\right)$ & $\left(\begin{array}{cc}1.90 & -1.82 \\
-1.82 & 2.06\end{array}\right)$ & $\left(\begin{array}{cc}2.13 & -2.01 \\
-2.01 & 2.27\end{array}\right)$ \\
\hline$r_{3}$ & -0.96 & -0.93 & -0.93 & -0.92 & -0.91 \\
\hline $\mathrm{E}$ & $15.33 \%$ & $12.67 \%$ & $14.22 \%$ & $10.67 \%$ & $11.33 \%$ \\
\hline
\end{tabular}

When faced to much more overlapping clusters (data set \#2), the parameter estimation process is strongly disturbed, the means being attracted by the dense areas, as shown in Table 4 As expected, robust estimates tends to correct this trend, i.e. the obtained values are closer to the theoretical ones (models \#3, \#4 and \#5). Consequently, the optimal Cauchy parameter values $c$ are smaller than those selected on data set \#1. Figure 3 shows the partitions that we have obtained with the best models involving robust estimates according to the parameter fitting and error as well (model \#4 on the left hand-side, model \#5 on the right hand-side).

Table 5. Results over the 50 runs

\begin{tabular}{|l|c|c|c|c|c|c|}
\hline & Error & Model \#1 & Model \#2 & Model \#3 & Model \#4 & Model \#5 \\
\hline \multirow{2}{*}{ Data set\#1 } & Mean & $8.31 \%$ & $9.87 \%$ & $9.86 \%$ & $11.87 \%$ & $2.96 \%$ \\
\cline { 2 - 7 } & StDev & 10.87 & 12.57 & 15.18 & 15.15 & 0.36 \\
\hline \multirow{2}{*}{ Data set\#2 } & Mean & $18.82 \%$ & $13.27 \%$ & $16.76 \%$ & $13.95 \%$ & $11.93 \%$ \\
\cline { 2 - 7 } & StDev & 5.8 & 4.27 & 5. & 6.97 & 0.2 \\
\hline
\end{tabular}


In order to test the robustness to initial center locations, we have chosen the optimal parameter setting with respect to the maximum posterior probability criterion. Table 5 summarizes the means and standard deviations of the error we have obtained over the 50 different runs. According to a mean value close to the minimum one and a low standard deviation, our model (\#5) outperforms all the others on both datasets. We think that the lower sensitivity of this model to initialization can be explained by the introduction of normal subcomponents that softens the tails of the resulting component. The ability of the model 5 to perform good clustering in spite of a bad initialization suggest us that it would be useful in many real situations.

\section{Conclusion}

In this paper, we have compared two different approaches to clustering multivariate data in the context of mixture of components likelihood maximization with the EM algorithm. Indeed, such algorithm often fails in finding accurate parameters when the data are mixed with noisy data. So, one can either take noisy data into account when defining the mixture model or use robust estimation techniques. We have noticed that both approaches can improve the results whatever the separability of the clusters is. Furthermore, in case of strong overlap, their joint use give better results. We have proposed such a model whose performances in terms of misclassification as well as accuracy of the parameters estimates are satisfactory. Moreover, we notice that this model is very robust to different initializations. Further investigation will concern the automatic selection of some coefficients involved in this model.
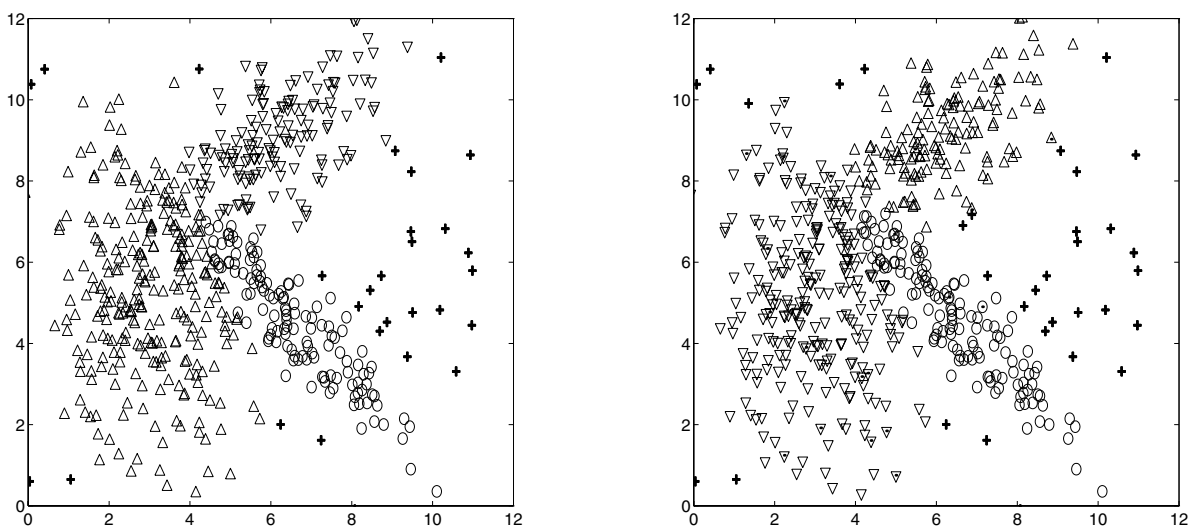

Fig. 3. Data set \#2 clustering with models \#4 (left) and \#5 (right) 


\section{Acknowledgements}

This work has been partially supported by the Conseil Général de CharenteMaritime.

\section{References}

1. Campbell, N.A., Lopuhad, H.P., Rousseeuw, P.J.: On the calculation of a robust S-estimator of a covariance matrix. Delft University of Technology. Tech. Report DUT-TWI-95-117 (1995)

2. Celeux, G., Soromenho, G.: An entropy criterion for assessing the number of clusters in a mixture model. J. of Classification 13 (1996) 195-212

3. Dempster, A.P., Laird, N.M., Rubin, D.B.: Maximum likelihood from incomplete data via the EM algorithm with discussion. J. of the Royal Stat. Soc. 39 (1977) $1-38$

4. McLachlan, G.J., Peel, D., Basford, K.E., and Adams, P.: The EMMIX software for the fitting of mixtures of normal and t-components. Journal of Statistical Software 4, No. 2. (1999).

5. Fraley, C., Raftery, A.E.: MCLUST: Software for model-based clustering and discriminant analysis. Univ. of Wash. Tech. Report TR-342 (1998)

6. Huber, P.J.: Robust statistics. John Wiley. New-York (1981)

7. Kharin, Y.: Robustness of clustering under outliers. LNCS 1280 (1997)

8. McLachlan, G.J., Peel, D.: Robust cluster analysis via mixtures of multivariate tdistributions. LNCS 1451 (1999) 658-667

9. Wu, C.F.J.: On the convergence properties of the EM algorithm. Ann. of Stat. 11 (1983) 95-103 